\title{
LITERATUR
}

\section{Lehren und Perspektiven aus 50 Jahren Integration}

\author{
Daniel Göler und Irene Kögl*
}

Der fünfzigste Jahrestag der Römischen Verträge Anfang 2007 und die Unterzeichnung des Lissabonner Vertrages am Ende desselben Jahres bieten einen willkommenen Anlass zur Rückschau auf den bisherigen Integrationsprozess wie zur Analyse der gegenwärtigen und künftigen Herausforderungen einer Gemeinschaft, die sich seit ihrer Gründung in Umfang und Charakter fundamentalen Veränderungen unterzogen hat. Die im Folgenden vorgestellten drei Sammelbände behandeln aus unterschiedlicher Perspektive die Erfahrung der bisherigen Integrationsgeschichte im Hinblick auf die aus ihr resultierenden Schlussfolgerungen für die künftige Gestalt der Europäischen Union.

Ausgangspunkt des Sammelbandes von Martin Koopmann und Stephan Martens ist die Krise, in der sich die Europäische Union nach dem Scheitern des Verfassungsvertrages befindet und die darin begründet gesehen wird, dass die Entscheidung für die Erweiterung auf ein Europa der 27 nicht überall auf gesellschaftlichen Konsens gestoßen ist. Daraus resultiert die zentrale Thematik des Bandes: Welches Europa wollen wir und welcher Gestalt sollte dieses Europa sein? ${ }^{1}$

Einen guten Überblick über die Kernthesen des Bandes gibt der Beitrag von Yves Bur und
Martin Koopmann/Stephan Martens (Hrsg.): Das kommende Europa. Deutsche und französische Betrachtungen zur Zukunft der Europäischen Union, Nomos: Baden-Baden 2008, ISBN 978-38329-3265-7; 388 Seiten, 49,00 €.

Christian Joerges/Matthias Mahlmann/Ulrich K. Preuß (Hrsg.): „Schmerzliche Erfahrungen der Vergangenheit" und der Prozess der Konstitutionalisierung Europas, VS Verlag: Wiesbaden 2008, ISBN 978-3-531-15414-5; 353 Seiten, $44,90 €$.

Wolf Schäfer/Andreas Graf Wass von Czege (Hrsg.): Das Gemeinsame Europa - viele Wege, kein Ziel?, Nomos: Baden-Baden 2007, ISBN 978-3-8329-2902-2; 304 Seiten, 58,00 €.

Andreas Schockenhoff. Trotz der positiven Bilanz, die die Autoren der Europäischen Union seit den Römischen Verträgen attestieren, ${ }^{2}$ bedürfe es mit zunehmender Erweiterung und Vertiefung des Integrationsprozesses nun allerdings einer öffentlichen Diskussion über eine europäische Identität: „Was macht die Europäische Union aus, worin bleibt sie sich treu, und was macht sie im permanenten Wandel wiedererkennbar?" ${ }^{3}$ In diesem Zusammenhang gelte es auch die Rolle des traditionellen deutsch-französischen Motors zu überdenken,

* Prof. Dr. Daniel Göler, Professor für European Studies, Universität Passau. Irene Kögl, Mitarbeiterin an der Professur für European Studies, Universität Passau.

1 Martin Koopmann/Stephan Martens: Einleitung, in: Martin Koopmann/Stephan Martens (Hrsg.): Das kommende Europa. Deutsche und französische Betrachtungen zur Zukunft der Europäischen Union, Baden-Baden 2008, S. 15-20, hier S. 16.

2 Yves Bur/Andreas Schockenhoff: Vorwort, in: Martin Koopmann/Stephan Martens (Hrsg.): Das kommende Europa. Deutsche und französische Betrachtungen zur Zukunft der Europäischen Union, Baden-Baden 2008, S. 11-13, hier S. 11.

3 Ebenda. 
der durch die frühzeitige Einbeziehung Dritter mehr Schubkraft gewinnen könnte. ${ }^{4}$

Zentral bei der Frage nach der europäischen Identität sind jene europäischen Werte, auf denen die Europäische Union sich gründet und welche zugleich entscheidend sind für eine Kohärenzbildung innerhalb des doch sehr heterogenen Staatengebildes, vor allem wenn argumentiert wird, dass das ,,[...] Zusammengehörigkeitsgefühl der Europäer [...] das Ergebnis eines Jahrhunderte währenden Kulturprozesses der Differenzierung wie der Vereinheitlichung" ist. ${ }^{5}$ Doch auch wenn Pluralität somit als nicht unerhebliche Kraft für das Erreichen des bisherigen Status quo interpretiert wird, so fordern die Autoren dennoch eine differenzierte und vor allem behutsame Problematisierung ihrer Grenzen. Dies führt zu Fragen nach den limitierenden Faktoren wie auch den real existierenden geografischen Grenzen der Erweiterung unter Berücksichtigung des Axioms, dass ,jede Erweiterung den Zusammenhalt der Union wahren ${ }^{\text {“6 }}$ solle. Die daraus resultierende These lässt sich wie folgt verkürzen: Eine Bilanz der europäischen Integration kann nur dann zukunftsweisende Resultate liefern, wenn sie eine Synopse aus den Erfolgen einer sukzessiven Vertiefung und Erweiterung sowie den Auswirkungen auf die emotionale Komponente des Integrationsprozesses, das ,europäische Wir-Gefühl`, bildet.

Das Fehlen eines solchen Wir-Gefühls thematisiert Reiner Marcowitz, der aus geschichtswissenschaftlicher Perspektive den Versuch unternimmt, sich dem Phänomen der derzeitigen Euroskepsis zu nähern. Aus dieser Aus- gangssituation heraus leitet Marcowitz die "Méthode Monnet" als das zentrale Bewegungsgesetz der europäischen Einigung ab. ${ }^{7}$ Die Vorgehensweise, durch Kollektivlösungen bei konkreten Problemen Anreize für Kompetenzübertragungen der Nationalstaaten zu schaffen, thematisiert Marcowitz unter Verweis auf die Akzeptanzprobleme des heutigen Integrationsprojektes. Die heutige Legitimationskrise nach dem Ende des ,permissive consensus', die durch das mangelnde Identifikationspotenzial des Elitenprojektes Europäische Union schrittweise entstand, bietet zugleich die Grundlage der Empfehlung des Autors: Die Europäische Union muss von einem breiten gesellschaftlichen Konsens getragen werden. ${ }^{8}$ Was auf gesellschaftlicher Ebene den besagten Konsens zu schaffen vermag und ,heute, im Namen der Solidarität, die Einheit der EU-Länder festigen kann", 9 dieser Frage widmet sich Stephan Martens in seinem Beitrag. Dabei greift er die Kritik von Marcowitz an der „Méthode Monnet“ auf: Diese habe versucht, den „Europäer peu à peu in ein Bündel aus solidarischen Verpflichtungen, wirtschaftlichen und politischen Mechanismen einzubinden, bis hin zur Schaffung einer unwiderruflichen Schicksalsgemeinschaft" ${ }^{10}$ Für Martens liegt der Grund des voranschreitenden Wirksamkeitsverlusts dieser pragmatisch-funktionalistischen Herangehensweise darin, dass der europäische Einigungsprozess mittlerweile Fragen staatlicher Souveränität berührt, deren Lösungen ein Mindestmaß an Zugehörigkeitsgefühl voraussetzen.

Genau dieses fehlende Zugehörigkeitsgefühl beziehungsweise das Fehlen einer gemeinsa-

4 Martin Koopmann: Auf der Suche nach dem verlorenen Gleichgewicht: Aspekte eines Neuanfangs deutschfranzösischer Zusammenarbeit in der Europa-Politik, in: Martin Koopmann/Stephan Martens (Hrsg.): Das kommende Europa. Deutsche und französische Betrachtungen zur Zukunft der Europäischen Union, BadenBaden 2008, S. 231-242, hier S. 231.

5 Bur/Schockenhoff: Vorwort, 2008, S. 11-12.

6 Ebenda, S. 12.

7 Reiner Marcowitz: 50 Jahre Römische Verträge: Rückblick und Ausblick, in: Martin Koopmann/Stephan Martens (Hrsg.): Das kommende Europa. Deutsche und französische Betrachtungen zur Zukunft der Europäischen Union, Baden-Baden 2008, S. 21-38, hier S. 25.

8 Ebenda, S. 37.

9 Stephan Martens: Europa, eine Schicksalsgemeinschaft?, in: Martin Koopmann/Stephan Martens (Hrsg.): Das kommende Europa. Deutsche und französische Betrachtungen zur Zukunft der Europäischen Union, BadenBaden 2008, S. 39-52, hier S. 39.

10 Ebenda, S. 51. 
men Identität steht im Zentrum des Sammelbandes von Christian Joerges, Matthias Mahlmann und Ulrich K. Preuß. Ausgangspunkt ist die Feststellung, dass trotz der vielfachen Betonung der historischen Erfahrungen in ,Sonntagsreden" die konkrete Antwort darauf, was die europäische Geschichte und Identität sein soll, zu kurz kommt und selbst in der Präambel des Verfassungsvertrages der Geschichtsbezug lediglich in der Formulierung der „schmerzlichen Erfahrungen“11 der Vergangenheit seinen Niederschlag fand.

Tenor der meisten Beiträge ist, dass sich mit der Osterweiterung das „Fehlen einer gemeinsamen Erinnerungskultur"12 noch verstärkt hat, was sich defizitär auf die immer tiefgreifendere Rechtsgemeinschaft auswirkt. Christian Joerges fragt deshalb, ob und wie sich überhaupt ein ,,acquis historique“ denken lasse, ${ }^{13}$ zumal es eine „Rechtsgeschichte des institutionalisierten Europa, in der die Europarechtsverständnisse der verschiedenen nationalen Rechtskulturen und deren interne Kontroversen rekonstruiert worden wären [...,] nicht" gibt. ${ }^{14}$ Deshalb kommt es darauf an, Methoden zu entwickeln, um europäisches Recht vor dem Hintergrund unterschiedlicher Rechtstraditionen zu interpretieren. Ziel wäre demnach nicht eine gemeinsame europäische Erinnerungskultur, sondern eine Bewusstmachung der Pluralität derselben.

Das Fehlen einer gemeinsamen Erinnerungskultur problematisiert auch Mattias Kumm in seinem Aufsatz „Geschichte als Argument?“, der sich um die Frage dreht, ,welche öffentliche Rolle Geschichte in einer durch [...] Prinzipien konstituierten Rechtsgemeinschaft spielen soll und welche Rolle ihr nicht zuzumuten ist“". ${ }^{15}$ Sein Schluss ist hierbei: ,Vergangenheit legitimiert nicht, weil sowohl wir als auch andere immer auch anders können, und deshalb zu prüfen haben, was wir tun sollen. "16 Damit weist er auf eine der Grundaussagen des gesamten Bandes hin: Die Geschichte oder die „schmerzlichen Erfahrungen der Vergangenheit" können die Europäische Union nicht legitimieren. Dies würde nur zu einer rückwärts gewandten Europa-Nostalgie führen, die allenfalls in der Gründergeneration, bei der die Erfahrungen von Krieg und Gewaltherrschaft noch unmittelbar präsent waren, dem Integrationsprozess einen Sinn geben konnte und die nach der Osterweiterung weiter an Tragfähigkeit verloren hat. Eine europäische Identität ist daher nur schwer aus der Geschichte abzuleiten, sondern ein Projekt der Zukunft, was Johannes Pollak in seinem Beitrag darauf zurückführt, „dass kollektive Identität nicht einfach aus Traditionen, gemeinsamer Sprache etc. abgeleitet werden kann, sondern dass sie politisch bestimmt werden muss“. ${ }^{17}$ „Die Europäische Union kann also eine kollektive Identität entwickeln [...]. [Dies] erfordert einen politischen Willen, der [jedoch] nicht absehbar ist“ ${ }^{18}$

Als mögliche identitätsstiftende Elemente werden in verschiedenen Beiträgen zwar das euro-

11 Siehe Amtsblatt der EU, Nr. C 310 vom 16. Dezember 2004, S. 3.

12 Vgl. unter anderem Konrad H. Jarausch: Konfligierende Erinnerungen. Nationale Prägungen, Verständigungsversuche und europäische Geschichtsbilder, in: Christian Joerges/Matthias Mahlmann/Ulrich K. Preuß (Hrsg.): „Schmerzliche Erfahrungen der Vergangenheit“ und der Prozess der Konstitutionalisierung Europas, Wiesbaden 2008, S. 15-25, hier S. 16.

13 Christian Joerges: Kollisionsrecht als Form der Konstitutionalisierung Europas nach „schmerzlichen Erfahrungen“: Zur Kritik der Geschichtsvergessenheit der Europawissenschaft, in: Christian Joerges/Matthias Mahlmann/Ulrich K. Preuß (Hrsg.): „Schmerzliche Erfahrungen der Vergangenheit“ und der Prozess der Konstitutionalisierung Europas, Wiesbaden 2008, S. 26-42, hier S. 27.

14 Ebenda, S. 31

15 Mattias Kumm: Geschichte als Argument? Republikanisches Geschichtsverständnis im Transnationalen Europa, in: Christian Joerges/Matthias Mahlmann/Ulrich K. Preuß (Hrsg.): ,Schmerzliche Erfahrungen der Vergangenheit“ und der Prozess der Konstitutionalisierung Europas, Wiesbaden 2008, S. 43-47, hier S. 43.

16 Ebenda, S. 45.

17 Johannes Pollak: Ist eine Europäische Identität möglich? Oder: Warum wir lernen sollten, Zwiebeln zu lieben, in: Christian Joerges/Matthias Mahlmann/Ulrich K. Preuß (Hrsg.): ,Schmerzliche Erfahrungen der Vergangenheit“ und der Prozess der Konstitutionalisierung Europas, Wiesbaden 2008, S. 63-80, hier S. 64-65.

18 Ebenda, S. 80 
päische Sozialstaatsmodell, der Menschenrechtsschutz, das Binnenmarktprojekt oder auch das Subsidiaritätsprinzip gesehen. Schwierig sind allerdings die divergierenden Konnotationen dieser Werte in den Mitgliedstaaten. So weist Stefan Oeter in seinem Beitrag auf die Problematik der unterschiedlichen historischen „Belastung“ der meisten staatsrechtlichen Kategorien hin, was sich insbesondere an dem Streit über eine bundesstaatliche Finalität zeigt. „Man sollte [... daher] aufhören, die ,real existierende' Europäische Union an Trugbildern eines idealen ,europäischen Bundesstaates' zu messen - und die Verfehlung dieses Ideals als nicht gutzumachende ,Erbsünde ' des Integrationsprozesses zu denunzieren." ${ }^{\text {19 }}$

Die Frage nach dem Ziel der Integration steht auch im Zentrum des Sammelbandes von Wolf Schäfer und Andreas Graf Wass von Czege. Anders als die beiden anderen Bände orientiert er sich in der Frage nach den Finalitäten der europäischen Integration weniger an einer künftigen europäischen Identität, sondern an der konkreten Politikgestaltung. Dabei konzentriert er sich vor allem auf die Konsequenzen der Osterweiterung sowie daraus abzuleitenden Herausforderungen für die vergemeinschafteten Politikfelder der Europäischen Union. Die korrespondierenden Beiträge deutscher und ungarischer Ökonomen und Juristen folgen der Prämisse, ein differenziertes und facettenreiches Bild der „Vielfalt und Vielschichtigkeit der Herausforderungen und Wege eines gemeinsamen Europa“ ${ }^{20}$ zu vermitteln.

Dem Verhältnis der Herausforderungen zu Beginn des Integrationsprozesses zum gegen- wärtigen Status quo widmet sich Gert Nicolaysen, der argumentiert, dass sich die Konzeption der Supranationalität als prinzipiellem Integrationsmodus aus dem Ziel der europäischen Gründungsväter ableiten lässt, die von den tradierten „Methoden des Friedensschlusses, des Gleichgewichts und der Hegemonie“21 abzugrenzen ist. In einem nächsten Schritt transferiert er das Konzept der Supranationalität in die Gegenwart und versucht, dessen Umsetzung vor dem Hintergrund der europäischen Verträge zu beurteilen, ohne jedoch den Rückbezug auf jene Zielsetzungen zu vernachlässigen, die das Entstehen des Terminus historisch bedingten. An die expliziten Defizite der Supranationalität innerhalb der institutionellen Strukturen der Europäischen Union schließt der Beitrag von Martin Seide $^{22}$ an. Vor allem um ein effizientes und effektives Policy-Making in den Bereichen der Wirtschafts-, Sozial- und Gesellschaftspolitik zu ermöglichen, hält er einen Ausbau der Union zu einer ,echten Föderation“ für unabdingbar.

Eine gelungene Synthese des zentralen Spannungsfeldes zwischen Erweiterung und Vertiefung findet sich im abschließenden Beitrag von Wolf Schäfer, der zugleich auch als Resümee des Sammelbandes angesehen werden kann: Zwischen Erweiterung und Vertiefung liege ein „substitutionales Verhältnis“ vor, wonach ,mit jeder Erweiterungsrunde grundsätzlich ein Schritt zu weniger Vertiefungspotenzial gegangen“" werde. ${ }^{23}$ Daraus lässt sich ein ,Trade-Off“ aus Skalenerträgen bei der Produktion öffentlicher Güter und den Kosten der Heterogenität ableiten unter der Voraus-

19 Stefan Oeter: Europäischer Föderalismus-Streit. Eine Unionsverfassung jenseits von Bundesstaat und Staatenbund?, in: Christian Joerges/Matthias Mahlmann/Ulrich K. Preuß (Hrsg.): ,Schmerzliche Erfahrungen der Vergangenheit" und der Prozess der Konstitutionalisierung Europas, Wiesbaden 2008, S. 185-202, hier S. 201.

20 Wolf Schäfer/Andreas Graf Wass von Czege: Vorwort, in: Wolf Schäfer/Andreas Graf Wass von Czege (Hrsg.): Das Gemeinsame Europa - viele Wege, kein Ziel?, Baden-Baden 2007, S. 5.

21 Gert Nicolaysen: Das Integrationskonzept der Gründungsverträge, in: Wolf Schäfer/Andreas Graf Wass von Czege (Hrsg.): Das Gemeinsame Europa - viele Wege, kein Ziel?, Baden-Baden 2007, S. 33-56, hier S. 44.

22 Martin Seidel: Konsolidierung und Reform der Europäischen Union, in: Wolf Schäfer/Andreas Graf Wass von Czege (Hrsg.): Das Gemeinsame Europa - viele Wege, kein Ziel?, Baden-Baden 2007, S. 87-114.

23 Wolf Schäfer: Die Europäische Union: Der trade-off zwischen Erweiterung und Vertiefung, in: Wolf Schäfer/ Andreas Graf Wass von Czege (Hrsg.): Das Gemeinsame Europa - viele Wege, kein Ziel?, Baden-Baden 2007, S. 287-300, hier S. 287. 
setzung heterogener Präferenzstrukturen der beteiligten Individuen. Schäfer argumentiert, dass die vorherrschende Strategie der Europäischen Union zu einer sukzessiven Erweiterung bei gleichzeitiger institutioneller Vertiefung eine Priorisierung der Skalenerträge impliziere und die zunehmenden Heterogenitätskosten vernachlässige. Diese Strategie bedinge aber eine fortschreitende Aneignung immer neuer Politikfelder, die bis dato im Kompetenzbereich der Mitgliedstaaten lagen. Welche Konsequenzen eine solche Ausweitung der Politikfelder für die Akzeptanz Europas bei den Bürgern und für die europäische Identitätsfrage hat, bleibt allerdings offen.

Das zentrale Argument aller drei Sammelbände kann darin gesehen werden, dass die europäische Integration seit ihren Anfängen vor mehr als 50 Jahren fundamentalen Veränderungen ausgesetzt war. Vor allem haben sich die Rahmenbedingungen der Integration sehr weitreichend durch die Prozesse der Erweiterung und Vertiefung verändert, sowohl im Hinblick auf die europäische Identitätsfrage, die sich heute in ganz anderer Dringlichkeit als vor 50 Jahren stellt und nach der Osterweiterung eine weitaus größere Komplexität aufweist als in den Gründungsjahren, als auch im Hinblick auf die effiziente Politikgestaltung. Europa muss dieser doppelten Herausforderung gerecht werden und adäquate Antworten finden. Worin diese bestehen könnten, zeigen die drei Sammelbände aus unterschiedlicher Perspektive auf, womit sie einen anerkennenswerten Beitrag für die Europaforschung leisten.

\section{Zehn Jahre ESVP}

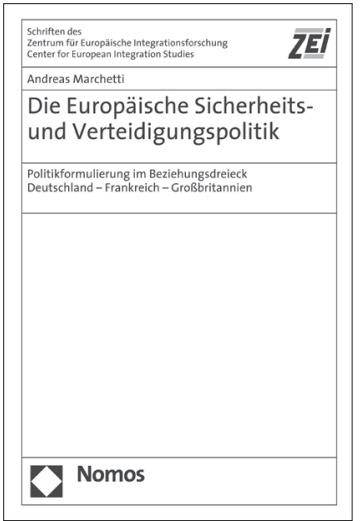

Bitte bestellen Sie im Buchhandel oder versandkostenfrei unter www.nomos-shop.de

\section{Die Europäische Sicherheits- und Verteidigungspolitik}

Politikformulierung im Beziehungsdreieck

Deutschland - Frankreich - Großbritannien

Von Dr. Andreas Marchetti

2009, 378 S., geb., 79,-€,

ISBN 978-3-8329-4530-5

(Schriften des Zentrum für Europäische

Integrationsforschung (ZEI), Bd. 70)

Die Studie behandelt die zentralen Fragen nach dem Warum und Wie der Integration der Sicherheits- und Verteidigungspolitik seit 1998. Der Autor zeigt, wie bisweilen sehr unterschiedliche nationale Motivationen die Modalitäten zur Ausformulierung der ESVP bedingen und überprüft, inwieweit die Effekte der ESVP mit den verlautbarten Motivationen übereinstimmen. 